\title{
Contribution de l'aérodynamique au développement durable et sécuritaire dans l'automobile
}

\author{
PATrick Gilliéron ${ }^{\mathrm{a}}$ ET EugÉnie Levallois-Goy \\ Direction de la Recherche, Renault SA, Service 64260, code API : TCR AVA 058, 1 avenue du golf, 78288 Guyancourt Cedex, \\ France
}

Reçu le 5 avril 2004, accepté le 5 décembre 2004

\begin{abstract}
Résumé - La contribution de l'aérodynamique pour le développement durable et sécuritaire dans l'automobile est présentée et argumentée à partir de travaux de recherche destinés à contrôler les écoulements pour réduire la consommation et améliorer la stabilité dynamique. Les résultats révèlent la complexité des phénomènes physiques et des problématiques à résoudre pour pouvoir agir sur les mécanismes d'apparition, de développement, d'interaction et de stabilité des structures tridimensionnelles décollées instationnaires. Cette complexité confirme la nécessité de développer des coopérations afin d'améliorer la compréhension des phénomènes physiques et rechercher des solutions qui permettent de contrôler des écoulements décollés associés ou non à des configurations transitoires.
\end{abstract}

Mots clés : Aérodynamique / développement durable / développement sécuritaire / contrôle / transitoire

\begin{abstract}
Contribution of the aerodynamic on the durable and safety development in automotive. The contribution of aerodynamics for the durable and safety development in automotive is presented and argued from research tasks intended to control the flows to reduce consumption and to improve dynamic stability. The results reveal the complexity of the physical phenomena and the problems to solve before being able to act on the appearance mechanisms, development, interaction and on the stability of the unsteady separated three-dimensional structures. This complexity confirms the need to develop co-operations in order to improve comprehension of the physical phenomena and to seek solutions which make it possible to control separated flows associated or not with transient configurations.
\end{abstract}

Key words: Aerodynamics / durable development / safety development / control / transient

\section{Introduction}

Les recherches entreprises aujourd'hui dans le domaine de l'aérodynamique appliquée à l'automobile s'effectuent dans la perspective du développement durable et sécuritaire.

Le développement durable suppose de mettre au point des techniques de contrôle des écoulements capables de réduire la consommation et les émissions de gaz à effet de serre. Ces réductions, fortement liées à une diminution de la traînée aérodynamique, nécessitent de modifier localement la trajectoire de l'écoulement, de supprimer ou de retarder la position des décollements ou encore de réduire le développement des structures tourbillonnaires décollées de sillage. En pratique, il s'agit de contrôler localement l'écoulement avec ou sans apport d'énergie à

\footnotetext{
a Auteur correspondant :

patrick.gillieron@renault.com
}

partir de dispositifs actifs ou passifs, Gilliéron [1]. Ces dispositifs, utilisés seuls ou en association, peuvent permettre de diminuer de manière significative la consommation des véhicules automobiles. Sur ce sujet, des analyses récentes montrent qu'en réduisant de $20 \%$ la traînée aérodynamique, il serait possible de réduire la consommation énergétique de $10 \%$. Au niveau de l'Europe, cette réduction de consommation serait de l'ordre de 24 milliards d'euros par an, Lesieur [2].

Le développement sécuritaire est associé aux objectifs d'amélioration de la sécurité des véhicules automobiles. Il s'agit ici de développer de nouvelles techniques capables d'une part de réduire les effets des phénomènes transitoires de type coup de vent latéral, dépassement ou croisement de deux véhicules, et d'autre part, de diminuer les distances d'arrêt lors des phases de freinage d'extrême urgence. La recherche de solutions nécessite alors de mettre au point des systèmes de contrôle des écoulements 


\section{Nomenclature}

\begin{tabular}{|ll|}
\hline$\vec{V}_{\mathrm{o}}:$ & Vecteur vitesse de l'écoulement incident \\
$V_{\mathrm{o}}:$ & Norme du vecteur vitesse $\vec{V}_{\mathrm{o}}$ en $\mathrm{m}_{\mathrm{s}}{ }^{-1}$ \\
$P_{\mathrm{io}}:$ & Pression d'arrêt associée à l'écoulement de vitesse $\vec{V}_{\mathrm{o}}$ en Pascal $(\mathrm{Pa})$ \\
$P_{\mathrm{i}}:$ & Pression d'arrêt locale au point de coordonnées $(x, y, z)$ en Pascal $(\mathrm{Pa})$ \\
$\rho:$ & Masse volumique en kg.m ${ }^{-3}$ \\
$C_{P \mathrm{i}}:$ & Coefficient de perte de pression d'arrêt \\
$L:$ & Longueur du véhicule en $\mathrm{m}$ \\
$l:$ & Largeur du véhicule en $\mathrm{m}$ \\
$L_{\mathrm{h}}:$ & Distance associée à l'évolution pseudo-harmonique des coefficients aérodynamiques \\
$V_{\mathrm{r}}:$ & Vitesse relative du véhicule dépassant par rapport au véhicule dépassé en $\mathrm{m}^{-1}$ \\
$f_{\mathrm{cv}}:$ & Fréquence caractéristique pour le coup de vent latéral en Hertz (Hz) \\
$f_{\mathrm{dp}}:$ & Fréquence caractéristique pour le dépassement en Hertz (Hz) \\
$f_{\mathrm{cr}}:$ & Fréquence caractéristique pour le croisement en Hertz (Hz) \\
$S_{\mathrm{cv}}:$ & Nombre de Strouhal pour le coup de vent latéral \\
$S_{\mathrm{dp}}:$ & Nombre de Strouhal pour le dépassement \\
$S_{\mathrm{cr}}:$ & Nombre de Strouhal pour le croisement \\
$x:$ & Direction du vecteur vitesse $\vec{V}_{\mathrm{o}}$ \\
$y:$ & Direction horizontale perpendiculaire à $x$ \\
$\Delta x:$ & Distance entre l'avant du véhicule dépassé et l'avant du véhicule dépassant en m \\
$\Delta y:$ & Espacement latéral entre les faces adjacentes des véhicules dépassant et dépassé en $\mathrm{m}$ \\
$\beta$ & $\quad$ Angle de dérapage en degré en ${ }^{\circ}$ \\
$C_{y}:$ & Coefficient aérodynamique d'effort latéral (suivant $y)$ \\
\hline
\end{tabular}

susceptibles de modifier les distributions pariétales de pression et d'accroître les appuis sur les roues arrières.

Chaque solution doit être facile à mettre en œuvre et peu coûteuse. Dans cette perspective, le groupe « Mécanique des Fluides et Aérodynamique » de Renault travaille sur des programmes internes de recherche et développe des coopérations avec des partenaires travaillant sur des thématiques connexes. L'objectif final de ces actions consiste à développer des systèmes qui répondent aux exigences automobiles en terme d'efficacité énergétique, de réglementation, de coût et de qualité.

\section{Résultats de la recherche}

Des exemples de contribution aux processus de déploiement des politiques de développement durable et sécuritaire de Renault sont ici successivement décrits et commentés.

\section{(i) Contribution à la politique de développement durable}

La contribution de l'aérodynamique à la politique de développement durable de Renault porte principalement sur la recherche de solutions destinées à réduire la traînée aérodynamique. Cette recherche nécessite de bien comprendre l'ensemble des mécanismes physiques associés aux processus instationnaires de formation, de développement et d'interaction des structures tridimensionnelles décollées qui apparaissent à la surface des véhicules automobiles.

Dans cette perspective, les expérimentations et les visualisations effectuées en canal hydraulique s'avèrent particulièrement intéressantes, Spohn et al. [3]. Les faibles

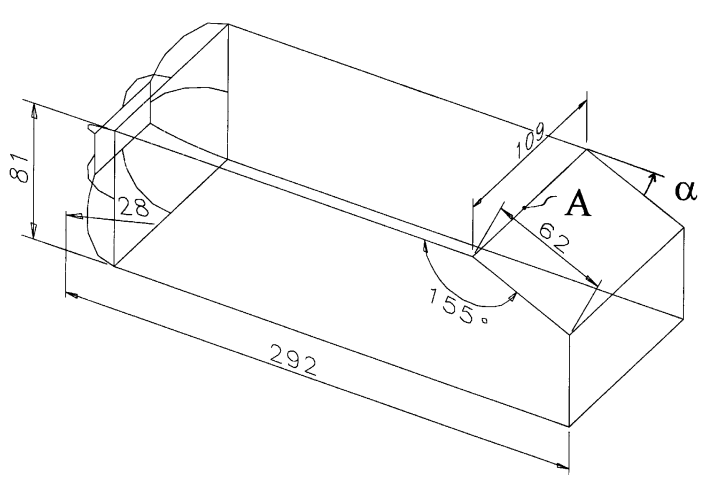

Fig. 1. Géométrie du « Corps de Ahmed » à l'échelle 1/5. Le repère $\mathrm{A}$ désigne l'arête de la fin du pavillon.

vitesses associées à l'utilisation du canal hydraulique permettent d'analyser les mécanismes de formation des structures tourbillonnaires, les interactions entre ces structures et d'effectuer des analyses fréquentielles à partir de simples enregistrements vidéo. Les enregistrements s'effectuent en lumière blanche ou à partir de plans laser et les phénomènes physiques peuvent être mis en évidence à partir de jets de fluorescéine ou de la décomposition électrolytique des rubans d'étain distribués sur la surface des maquettes.

Les résultats obtenus sur des géométries simplifiées pour de faibles nombres de Reynolds sont utilisés pour améliorer la compréhension des phénomènes physiques rencontrés à plus grand nombre de Reynolds et mettre au point des stratégies de contrôle.

À titre d'exemple, des expérimentations réalisées sur une géométrie simplifiée à arrière corps de type berline à échelle $1 / 15$, désignée corps de Ahmed ([4], voir Fig. 1), 


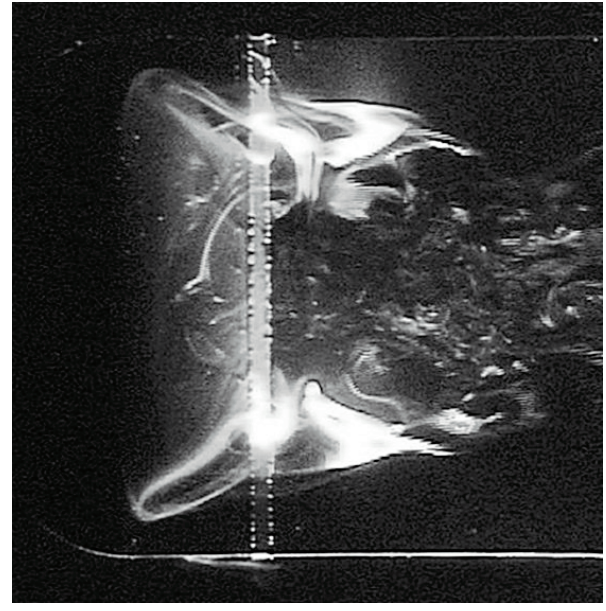

Fig. 2. Tomographie laser relevée dans un plan horizontal ( $z=$ const.) situé à $3 \times 10^{-3} \mathrm{~m}$ au-dessus du pavillon, vue du dessus du corps de Ahmed décrit figure 1 .

ont récemment permis d'enrichir la compréhension des phénomènes physiques associés aux processus de décollement qui peuvent apparaître sur le pavillon et la lunette arrière des véhicules automobiles.

Sur la partie avant, les tomographies relevées dans un plan horizontal parallèle au pavillon montrent une région décollée ouverte vers l'aval dans laquelle coexistent deux systèmes tourbillonnaires contrarotatifs [3], (Fig. 2). À ce résultat classique, l'analyse des images enregistrées sur bandes SVHS montre que le volume de la région décollée augmente transversalement pour se vider en partie à intervalles de temps réguliers vers l'aval. Ce phénomène se reproduit identique à lui-même à une fréquence proche de 0,21 Hertz. En considérant que ce phénomène physique ait même nombre de Strouhal à échelle 1, la fréquence des séquences de remplissage et de vidange de la région décollée est proche de 15 Hertz, [3]. Des visualisations relevées dans le plan médian longitudinal et vertical $(y=0)$ font de plus apparaître des instabilités de type Kelvin-Helmholtz dont la fréquence déterminée pour un véhicule à échelle grandeur est proche de $236 \mathrm{Hertz}$, (Fig. 3). Au décollement de pavillon sont donc associés des phénomènes basse fréquence pour le remplissage et la vidange de la région de recirculation et des phénomènes à fréquence plus élevée pour l'instabilité de cisaillement qui apparaît à la frontière entre la région décollée et l'écoulement externe.

Des résultats particulièrement intéressants peuvent également être mis en évidence sur la partie arrière. Ainsi, l'analyse des lignes de frottement relevées sur les parties latérales en amont de la fin du pavillon montre que l'écoulement pariétal latéral remonte vers le pavillon en amont de l'arête repérée A sur la figure 1, (écoulement latéral, Fig. 4). Cet écoulement alimente d'une part la structure tourbillonnaire longitudinale en cornet (cornet A, Fig. 4) issue du décollement latéral de lunette arrière et d'autre part le mouvement tourbillonnaire de rotation (cornet $\mathrm{B}$, Fig. 4) associé à la région décollée centrale D. Ce mouvement de fluide confirme l'existence

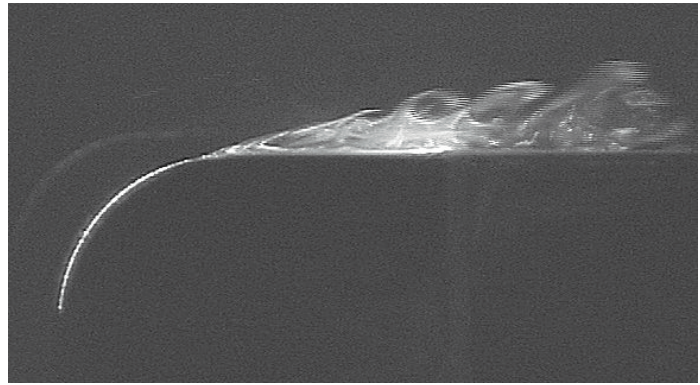

Fig. 3. Tomographie laser relevée dans le plan médian longitudinal $(y=0)$. Visualisation des structures décollées de Kelvin-Helmholtz.

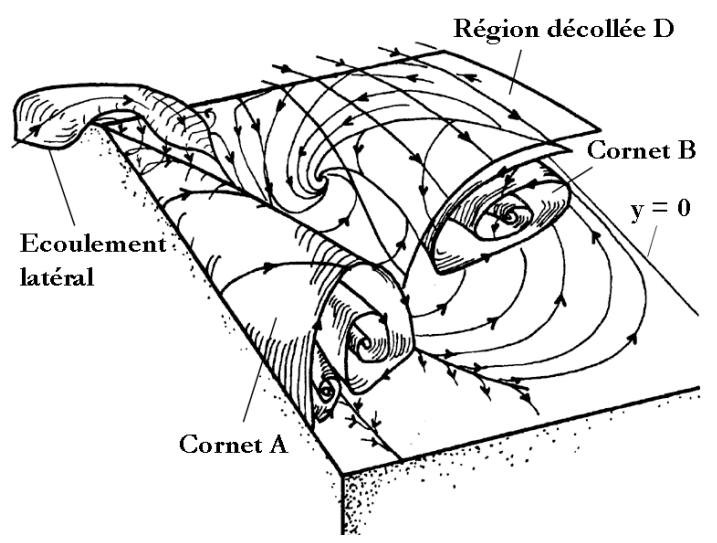

Fig. 4. Représentation topologique schématique de la structure décollée de la partie gauche de la lunette arrière, vue de l'arrière.

d'une région d'attachement mise en évidence par Gilliéron et al. [5] au voisinage des parties hautes et latérales de lunette arrière pour des nombres de Reynolds proches de $4,2 \times 10^{6}$.

L'ensemble de ces résultats s'avère particulièrement intéressant pour imaginer et mettre au point des stratégies de contrôle destinées à réduire la consommation et les émissions de polluants. Des actions sont en cours à la Direction de la Recherche de Renault et des partenariats forts pourraient être engagés avec les unités de recherche des secteurs aéronautiques.

Ces résultats ne prennent cependant pas en compte les évolutions topologiques transitoires des écoulements qui peuvent apparaître sur un véhicule dans des conditions réelles de circulation. Ainsi les conditions aux limites, généralement associées aux essais en soufflerie ou en canal hydraulique, sont invariables et ne permettent pas d'analyser les influences des évolutions spatiales et temporelles des champs de vitesses ou de pressions qui apparaissent lors d'un coup de vent latéral ou lors des processus de dépassement ou de croisement de deux véhicules. Afin d'estimer ces influences sur la structure de l'écoulement, des expérimentations sont réalisées sur une géométrie simplifiée constituée d'un corps cylindrique muni d'un demi ovale de Rankine à l'avant et d'un culot incliné à $43^{\circ}$ à l'arrière, Gilliéron et al. [6]. Une description succincte du 


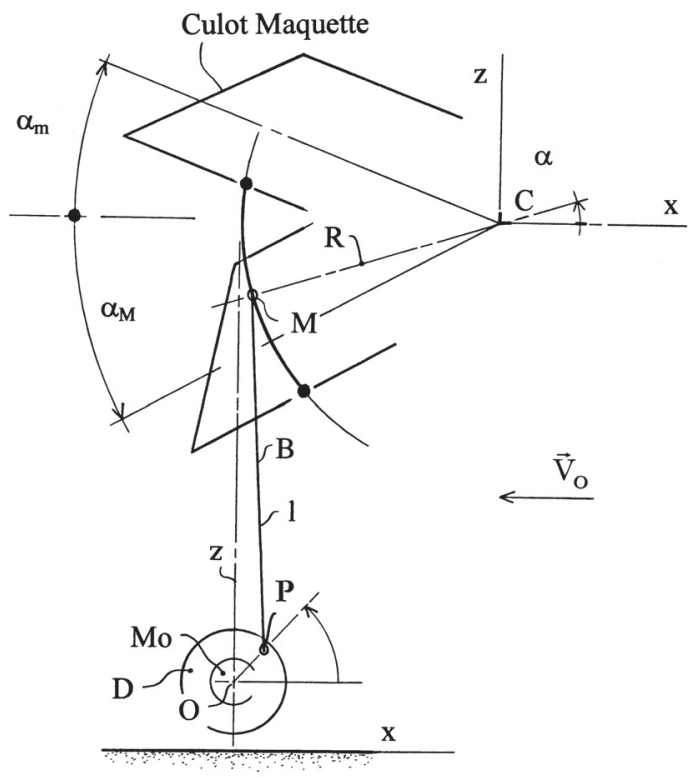

Fig. 5. Dispositif expérimental et partie arrière de la maquette repérée «Culot Maquette », d'après [6].

dispositif expérimental est donnée figure 5 (voir [6] pour plus de précisions).

La maquette peut osciller autour d'un axe horizontal $\mathrm{C}$ perpendiculaire à l'axe du cylindre et des montages adaptés permettent d'effectuer des expérimentations stationnaires et transitoires. Lors des essais stationnaires, la partie arrière de la maquette est reliée en $\mathrm{M}$ au plancher de la soufflerie par un mât vertical réglable en hauteur. Cette disposition permet de faire varier l'incidence $\alpha$ de la maquette de $\alpha_{\mathrm{m}}$ à $\alpha_{\mathrm{M}}$, (Fig. 5). Lors des essais transitoires, le mât est remplacé par un système comprenant un moteur Mo et un système bielle manivelle constitué du disque manivelle $\mathrm{D}$ de centre $\mathrm{O}$ et de la bielle $\mathrm{B}$. La maquette est alors animée d'un mouvement oscillant autour de l'axe $\mathrm{C}$ à une fréquence qui peut varier entre 0,5 et 2 Hertz. À chaque instant de date $t$, le point $\mathrm{M}$ se trouve à l'intersection des cercles de centres $\mathrm{P}$ et $\mathrm{C}$ et de rayons $l$ et $R$.

Des visualisations pariétales et des mesures de traînée aérodynamique permettent de caractériser les topologies stationnaires caractéristiques de l'écoulement pour différents angles d'incidence $\alpha$, figure 5. L'analyse des sillages stationnaires et instationnaires s'effectue ensuite à partir de tomographies de pertes de pression d'arrêt relevées dans le sillage à partir de plans parallèles et perpendiculaires à la direction de l'écoulement incident, Chometon et al. [7]. Ces pertes de pression d'arrêt, ho-

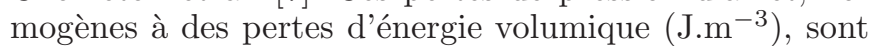
rapportées à la pression cinétique de l'écoulement incident et caractérisées par un coefficient de perte de pression d'arrêt $C_{P_{\mathrm{i}}}$ défini par :

$$
C_{P_{i}}=\frac{P_{\mathrm{i}_{\mathrm{o}}}-P_{\mathrm{i}}}{\frac{\rho}{2} V_{\mathrm{o}}^{2}}
$$

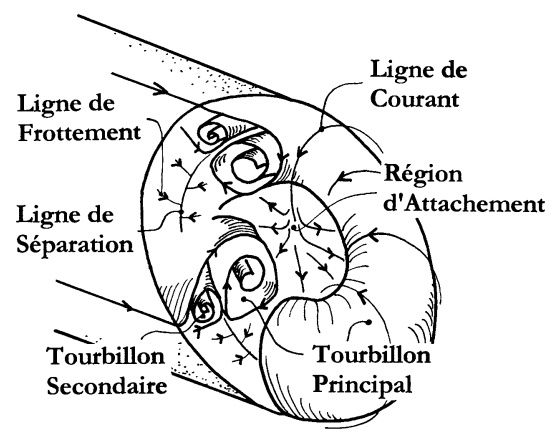

Fig. 6. Structure topologique caractéristique d'un sillage de type bidimensionnel (2D), $\alpha<\alpha_{\mathrm{c}}$ avec $\alpha_{\mathrm{c}}$ l'angle d'incidence critique relevé en stationnaire, [6].

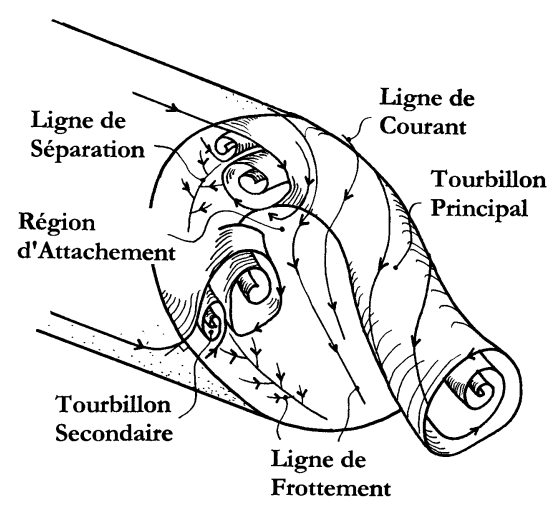

Fig. 7. Structure topologique caractéristique d'un sillage de type tridimensionnel (3D), $\alpha>\alpha_{\mathrm{c}}$ avec $\alpha_{\mathrm{c}}$ l'angle d'incidence critique relevé en stationnaire, [6].

où $P_{\mathrm{i}_{\mathrm{o}}}$ désigne la pression d'arrêt de l'écoulement incident de vitesse $V_{\mathrm{o}}, P_{\mathrm{i}}$ la pression d'arrêt locale de l'écoulement dans le sillage et $\rho$ la masse volumique de l'air.

La caractérisation des structures de sillage s'effectue alors en comparant les coefficients de traînée aérodynamique et les maximums des coefficients de pertes de pression d'arrêt relevés dans les mêmes plans de mesure. Toutes les expérimentations s'effectuent pour un nombre de Reynolds, calculé à partir de la longueur de la maquette, égal à $10^{6}$.

En stationnaire (fréquence nulle d'oscillation), le passage d'une structure tourbillonnaire bidimensionnelle 2D (Fig. 6) à une structure tridimensionnelle 3D (Fig. 7) s'effectue de manière brutale au voisinage d'un angle d'incidence critique $\alpha_{\mathrm{c}}$. Les impacts de l'évolution de ces structures sur les valeurs des coefficients de traînée aérodynamique et les maximums de pertes de pression d'arrêt sont mis en évidence figures 8 et 9, (Fig. 9 voir résultats sans oscillation, maquette fixe).

En régime transitoire, le passage d'une structure bidimensionnelle 2D (resp. 3D) à une structure tridimensionnelle 3D (resp. 2D) s'effectue de manière continue et l'évolution des maximums des pertes de pression d'arrêt relevés dans les sillages en fonction de l'incidence permet de mettre en évidence des phénomènes de déphasage et d'hystérésis [6], figure 9. Les résultats sont fonctions des valeurs des angles $\alpha_{\mathrm{m}}$ et $\alpha_{\mathrm{M}}$ pour $\alpha_{\mathrm{m}}<\alpha_{\mathrm{c}}<\alpha_{\mathrm{M}}$, 


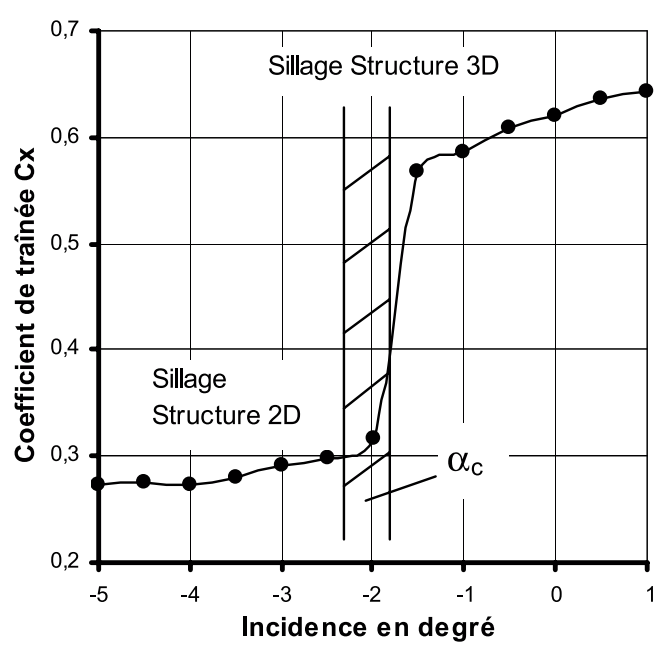

Fig. 8. Évolution des coefficients de traînée aérodynamique relevés sur la maquette fixe (sans oscillation) en fonction de l'angle d'incidence en degrés. La région hachurée contient l'angle d'incidence critique $\alpha_{\mathrm{c}}$.

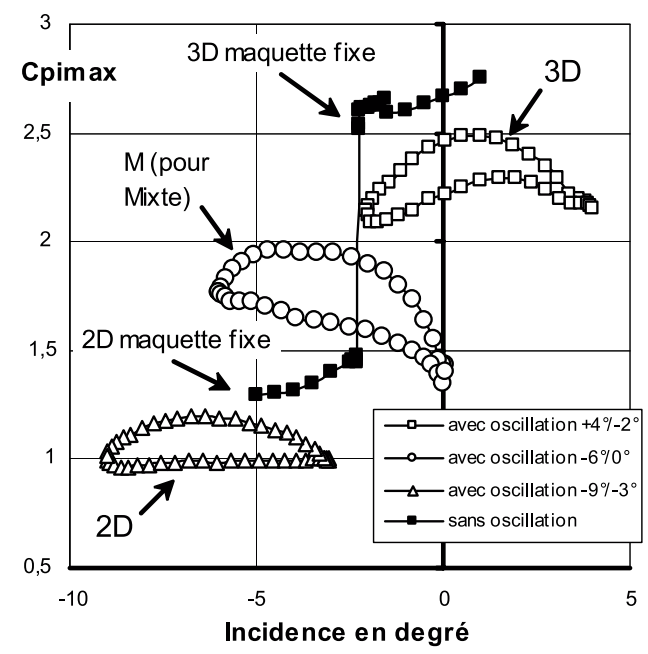

Fig. 9. Évolution des coefficients de pertes de pression d'arrêt maximums $C p_{\text {imax }}$ relevés dans le sillage à $100 \mathrm{~mm}$ en aval de la pointe arrière du culot et au centre des structures tourbillonnaires de sillage pour des positions fixes et oscillantes $(f=1 \mathrm{~Hz})$ en fonction de l'incidence. Les valeurs en degrés figurant dans l'encadré sont respectivement associées aux incidences mini et maxi repérées $\alpha_{\mathrm{m}}$ et $\alpha_{\mathrm{M}}$ figure 5 .

de l'amplitude angulaire $\alpha_{\mathrm{M}}-\alpha_{\mathrm{m}}$ et de la fréquence $f$ du mouvement d'oscillation de la maquette. La structure de sillage peut ainsi rester bidimensionnelle (2D) ou tridimensionnelle (3D) ou encore évoluer d'une structure 2D à une structure 3D ou inversement (structure mixte repérée M sur la Fig. 9). Dans ce dernier cas, les maximums des coefficients de pertes de pression d'arrêt tendent vers des valeurs caractéristiques d'un sillage bidimensionnel (2D) et la traînée aérodynamique reste faible. Ce résultat se déduit des corrélations relevées entre les coefficients de pertes de pression d'arrêt et les coefficients de traînée pour des configurations stationnaires, figures 8 et 9 .
Tous ces résultats suggèrent que les structures tourbillonnaires de sillage relevées en aval d'un véhicule automobile peuvent évoluer d'une structure 2D (resp. 3D) à une structure 3D (resp. 2D) lors d'un phénomène transitoire. L'influence de ces évolutions sur la traînée aérodynamique et la stabilité dynamique des véhicules automobiles reste à analyser et à quantifier. Cette problématique constitue un axe majeur de recherche dont les résultats pourraient montrer l'intérêt de mettre au point des systèmes capables de fixer ou de faire évoluer la topologie des sillages en fonction des conditions extérieures ou d'utilisation des véhicules.

\section{(ii) Contribution à la politique de développement sécuritaire}

S'agissant de contribuer à la politique de développement sécuritaire de Renault, le groupe «Mécanique des Fluides \& Aérodynamique » de la Direction de la Recherche de Renault travaille sur l'analyse des phénomènes transitoires de type coup de vent latéral, dépassement et croisement de deux véhicules. Ces processus transitoires s'accompagnent d'évolutions rapides de la topologie des sillages et des champs pariétaux de pression. Les véhicules sont alors soumis à des mouvements autour des axes de lacet, de roulis et de tangage qui peuvent avoir des incidences significatives sur la stabilité dynamique, le confort et la sécurité des véhicules.

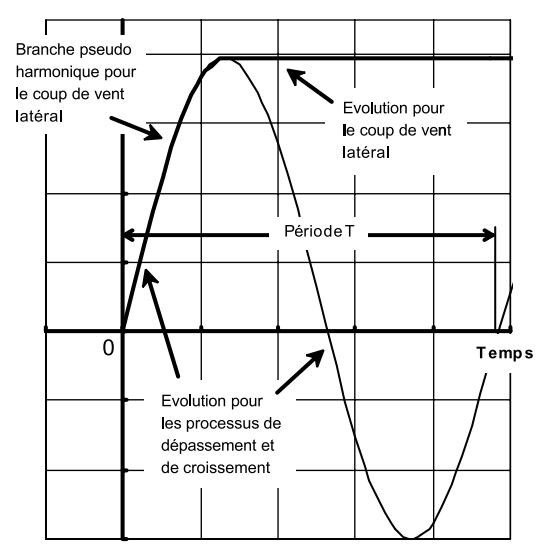

Fig. 10. Modèles d'évolution des coefficients aérodynamiques en fonction du temps pour les processus transitoires de type coup de vent latéral, dépassement et croisement de deux véhicules.

Les résultats publiés sur ces sujets et relevés dans la bibliographie restent cependant très incomplets pour constituer une source d'informations réellement satisfaisante pour l'industrie et les ingénieurs sont souvent démunis pour évaluer les effets des phénomènes transitoires.

Des approches analytiques basées sur l'hypothèse d'une évolution pseudo-harmonique des coefficients aérodynamiques ont alors été développées pour caractériser et quantifier ces phénomènes transitoires, figure 10. Chaque approche est élaborée en identifiant 


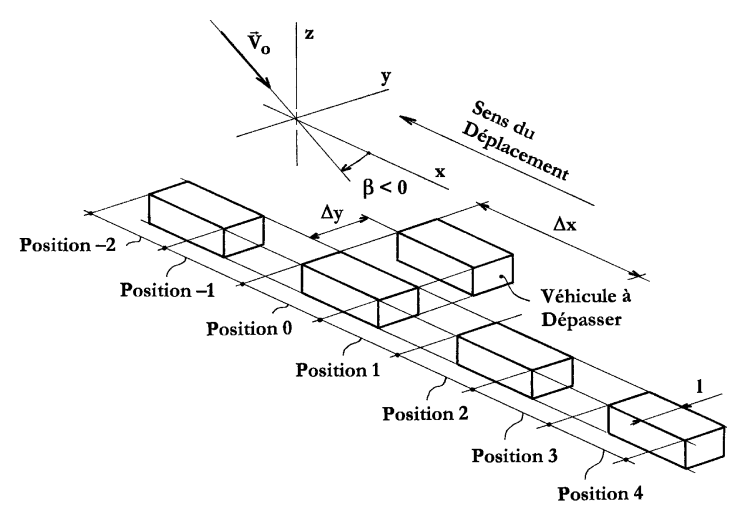

Fig. 11. Paramètres géométriques et positions longitudinales des véhicules dépassant et dépassé. Les positions $x / L=-3$ et -4 ne sont pas représentées.

une distance $L_{\mathrm{h}}$ associée à une période $T$ durant laquelle l'évolution de chaque coefficient aérodynamique est considérée comme harmonique ou pseudo-harmonique, figures 10 et 12 . Pour chaque processus transitoire sont alors associés une fréquence d'évolution $f$ et un nombre de Strouhal $S$ défini par rapport à la longueur $L$ du véhicule analysé. Les expressions analytiques des fréquences et des nombres de Strouhal ainsi obtenus pour le coup de vent latéral (cv) et les processus de dépassement ( $\mathrm{dp}$ ) et de croisement $(\mathrm{cr})$ sont respectivement données par Gilliéron et al. [7] :

$$
\begin{gathered}
f_{\mathrm{cv}}=\frac{V_{\mathrm{o}}}{4 L} \quad f_{\mathrm{dp}}=\frac{V_{\mathrm{r}}}{L_{\mathrm{h}}} \quad f_{\mathrm{cr}}=\frac{2 V_{\mathrm{o}}}{L_{\mathrm{h}}} \\
S_{\mathrm{cv}}=\frac{f L}{V_{\mathrm{o}}} \quad S_{\mathrm{dp}}=\frac{L}{L_{\mathrm{h}}} \quad S_{\mathrm{cr}}=\frac{f_{\mathrm{cr}} L}{2 V_{\mathrm{o}}}
\end{gathered}
$$

où $V_{\mathrm{O}}$ désigne la vitesse de déplacement du véhicule et $V_{\mathrm{r}}$ la vitesse relative du véhicule dépassant par rapport au véhicule dépassé. En pratique, les simulations numérique et expérimentale du coup de vent latéral et de croisement de deux véhicules étant particulièrement délicates à mettre en œuvre, la validation des hypothèses et des résultats analytiques s'effectue à partir de l'étude numérique du processus de dépassement.

Le processus de dépassement est alors analysé à partir de positions fixes des maquettes des véhicules dépassant et dépassé, Gilliéron [8]. Les trajectoires des deux véhicules sont parallèles, rectilignes et les coefficients aérodynamiques sont déterminés par rapport au référentiel $(x, y, z)$ où l'axe $x$ et le déplacement ont même direction, figure 11. Les résultats, obtenus à partir de géométries simplifiées de type corps de Ahmed (voir Fig. 1), valident l'hypothèse d'une évolution pseudoharmonique des coefficients aérodynamiques et les grandeurs fréquentielles déduites de l'approche analytique (voir Fig. 12 et Tab. 1). Cette validation consolide alors les ordres de grandeurs des fréquences et des nombres de Strouhal déterminés à partir des relations (2) et (3) pour le croisement et la sortie de tunnel en présence de vent latéral, [7].

Les résultats, reportés dans le tableau 1 et la figure 12 , ne prennent cependant pas en compte le caractère

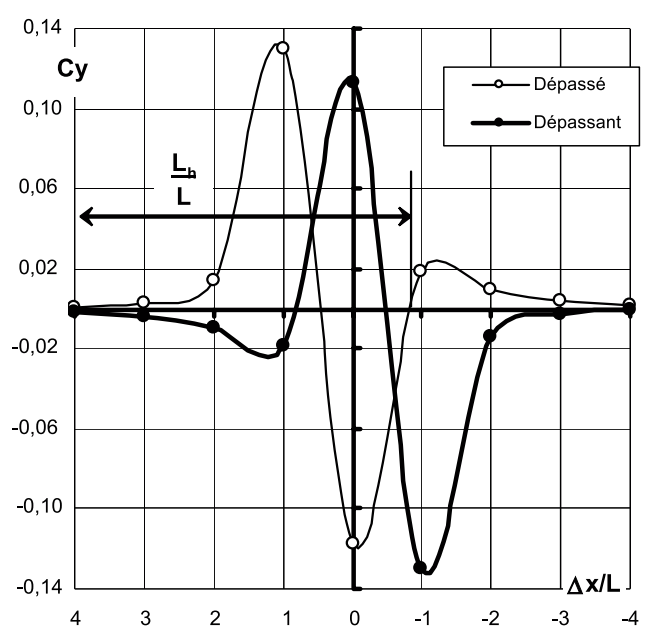

Fig. 12. Évolutions des coefficients aérodynamiques d'efforts latéraux des véhicules dépassé et dépassant en fonction de l'espacement longitudinal algébrique $\Delta x / L$ où $\Delta x$ désigne la distance entre l'avant du véhicule dépassé et l'avant du véhicule dépassant. Les résultats sont obtenus pour des positions fixes des véhicules dépassant et dépassé.

Tableau 1. Valeurs obtenues pour un véhicule de longueur $L=5 \mathrm{~m}$, une vitesse de déplacement $V_{\mathrm{o}}$ égale à $30 \mathrm{~m} \cdot \mathrm{s}^{-1}$ et une vitesse relative de déplacement $V_{\mathrm{r}}$ égale à $10 \mathrm{~m} \cdot \mathrm{s}^{-1}$.

\begin{tabular}{lcc}
\hline & Fréquence $f$ (Hertz) & Strouhal $S$ \\
\hline Sortie tunnel & 1,50 & 0,25 \\
Dépassement & 0,40 & 0,20 \\
Croisement & 2,40 & 0,20 \\
\hline
\end{tabular}

transitoire du processus de dépassement. Pour y remédier, un banc expérimental statique et dynamique de mesures et d'analyses du processus de dépassement a été développé en partenariat avec l'Institut Aérotechnique de Saint-Cyr l'École. Ce banc permet, d'une part, de valider les résultats analytiques et numériques, et d'autre part, de caractériser l'impact réel du processus transitoire sur les valeurs des efforts et des moments aérodynamiques. Il permet de simuler le processus dynamique de dépassement de deux véhicules pour différents espacements latéraux, différents angles de dérapage $\beta$ (Fig. 11) et des vitesses relatives de dépassement $V_{\mathrm{r}}$ comprises entre 0 et $10 \mathrm{~m} . \mathrm{s}^{-1}$. Une description détaillée du banc expérimental est donnée par Noger et al. [10].

La comparaison des résultats expérimentaux et numériques relevés pour une valeur nulle de la vitesse relative de dépassement confirme l'hypothèse de l'évolution pseudo-harmonique des coefficients d'efforts et de moments aérodynamiques ainsi que les ordres de grandeurs pour la fréquence et le nombre de Strouhal déduits de l'approche analytique, [7]. À titre d'exemple, les évolutions des coefficients aérodynamiques d'effort latéral obtenus par calculs et sur banc pour les véhicules dépassé et dépassant sont reportées figure 13 .

Les effets transitoires sur l'évolution du torseur aérodynamique sont ensuite analysés en faisant varier la vitesse relative de dépassement $V_{\mathrm{r}}$ et la distance $\Delta y$ de 


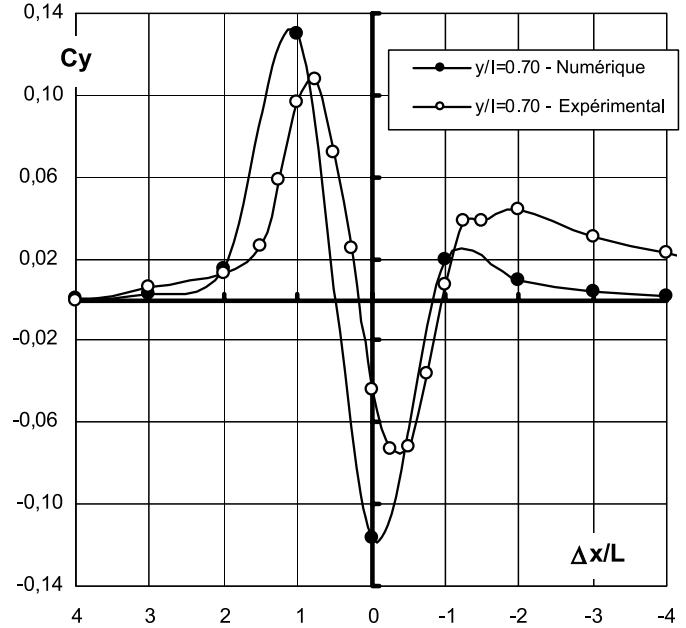

Fig. 13. Comparaison des coefficients aérodynamiques d'effort latéral mesurés sur banc et déterminés par calcul pour des positions fixes des véhicules dépassant et dépassé, $V_{\mathrm{r}}=$ $0 \mathrm{~m} . \mathrm{s}^{-1}$.

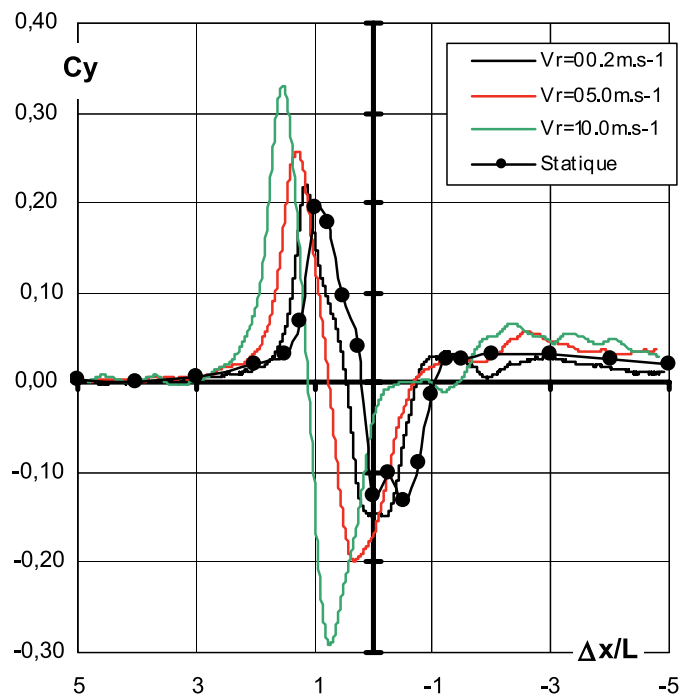

Fig. 14. Évolutions des coefficients aérodynamiques d'efforts latéraux appliqués sur le véhicule dépassé pour différentes vitesses relatives de dépassement et un espacement $\Delta y / l=0,25$. Notation : statique pour $V_{\mathrm{r}}=0 \mathrm{~m} \cdot \mathrm{s}^{-1}$.

séparation transversale entre les véhicules dépassant et dépassé, figure 11. Les évolutions des efforts et des moments aérodynamiques restent harmoniques quelle que soit la configuration. À titre d'exemple, les évolutions des coefficients aérodynamiques d'effort latéral $C_{y}$ relevés en faisant varier les espacements longitudinal $\Delta x$ et transversal $\Delta y$ entre les maquettes des véhicules dépassant et dépassé, sont reportées figures 14 et 15, [7] et [8]. Les résultats montrent que l'augmentation de la vitesse relative de dépassement accroît la fréquence d'évolution et l'amplitude des efforts tandis que la réduction de l'espacement transversal augmente l'amplitude mais reste sans effet sur la fréquence d'évolution. Pour l'espacement latéral réduit $\Delta y / l=0,25$, le rapport des efforts latéraux

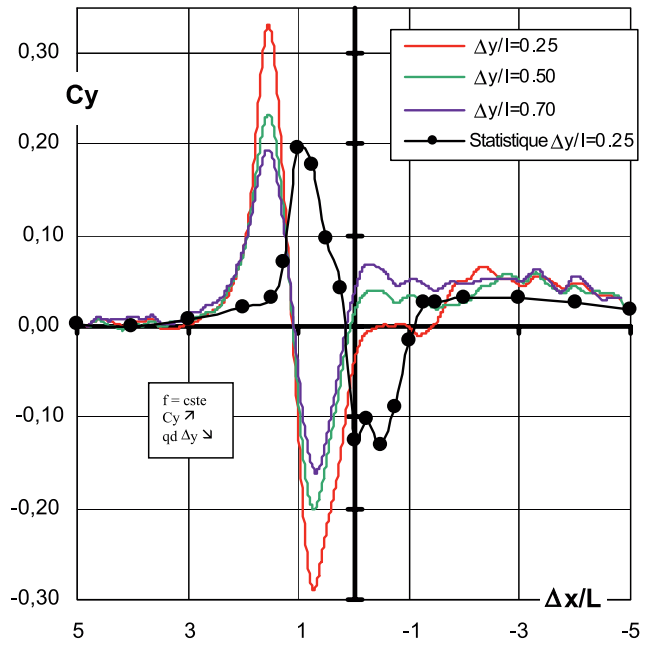

Fig. 15. Évolutions des coefficients aérodynamiques d'efforts latéraux appliqués sur le véhicule dépassé pour différents espacements transversaux réduits $\Delta y / l$, où $l$ désigne la largeur des maquettes supposées identiques des véhicules dépassé et dépassant. Les résultats, mesurés pour la vitesse relative de dépassement $V_{\mathrm{r}}=10 \mathrm{~m} \cdot \mathrm{s}^{-1}$, sont comparés aux résultats relevés pour des positions fixes des véhicules dépassé et dépassant. Notations : statique pour $V_{\mathrm{r}}=0 \mathrm{~m} \cdot \mathrm{s}^{-1}$.

maximums relevés pour les vitesses de dépassement $V_{\mathrm{r}}=$ 10 et $0 \mathrm{~m} . \mathrm{s}^{-1}$ est proche de 2,2 .

Tous ces résultats fournissent des informations qui permettent d'imaginer des stratégies de contrôle pour réduire les effets transitoires et améliorer la stabilité dynamique des véhicules automobiles. Leur élaboration nécessite cependant de travailler sur les actionneurs et les lois de contrôle. Des travaux sont en cours sur chacun de ces sujets.

\section{Conclusion}

La contribution de l'aérodynamique au développement durable et sécuritaire dans l'automobile est ici succinctement présentée et argumentée à partir de travaux de recherche effectués en canal hydraulique et en soufflerie.

Les expérimentations réalisées en canal hydraulique à partir d'une maquette fixe de géométrie simplifiée montrent la complexité topologique et instationnaire des phénomènes physiques à analyser et des problématiques à résoudre. Les expérimentations réalisées en souffleries à partir de maquettes mobiles confirment quant à elles la nécessité de prendre en compte et d'agir sur les phénomènes transitoires.

Tous les résultats montrent l'importance de développer la recherche pour d'une part améliorer la compréhension des processus d'apparition, de stabilité et d'interaction des structures décollées instationnaires et d'autre part rechercher des solutions qui permettent de contrôler l'apparition, l'évolution et le développement de ces structures. 
Pour Renault, l'objectif consiste à mettre au point des systèmes capables de réduire la consommation et d'améliorer la stabilité dynamique des véhicules automobiles. La rapidité d'obtention de ces solutions, associée à la nécessaire diminution des coûts de mise au point des différents systèmes qui pourraient être retenus pour contrôler les écoulements, nécessite cependant de développer des partenariats efficaces entre les chercheurs ayant à résoudre des problématiques identiques ou connexes. Sur ce point, la recherche partagée entre laboratoires ainsi que le développement des programmes du PREDIT et des Centres Nationaux de Recherche Technologiques (CNRT) constituent des opportunités pour les acteurs de la recherche scientifique qui doivent être encouragées et poursuivies.

\section{Références}

[1] P. Gillieron, Contrôle des Écoulements Appliqué à l'Automobile, État de l'Art, Mécanique \& Industries, décembre 2002

[2] M. Lesieur, La Turbulence, Collection Grenoble Sciences, Presses Universitaires de Grenoble, 1994, 184

[3] A. Spohn, P. Gillieron, Flow Separations Generated by a Simplified Geometry of an Automotive Vehicle,
Congress IUTAM Symposium on Unsteady Separated Flows, April 8-12, 2002, Toulouse, France

[4] S.R. Ahmed, R. Ramm, G. Faltin, Some salient features of the time-averaged ground vehicle wake SAE technical Paper Series 840300, Detroit 1984

[5] P. Gillieron, F. Chometon, Modelling of stationary three-dimensional detached airflows around an Ahmed Reference Body, Third International Workshop on Vortex, ESAIM, Proceedings, 7, 1999, pp. 173-182, http://www. emath.fr/proc/Vol7/

[6] P. Gillieron, F. Chometon, J. Laurent, Analysis of Hysteresis and Phase Shifting Phenomena in Unsteady Three-Dimensional Wakes, Experiments in Fluid 35, 117129, DOI 10.1007/S00348-002-0555-z, July 2003

[7] P. Gillieron, C. Noger, Contribution to the Analysis of Transient Aerodynamic Effects Acting on Vehicles, SAE paper, 2004, à paraître

[8] P. Gillieron, Detailed Analysis of the Overtaking Process, PREDIT2 Project, Unsteady Aerodynamics of Ground Vehicles and their Environment, J. Mech. Eng., January 2003

[9] C. Noger, P. Gillieron, Banc d'analyse du processus transitoire de dépassement de deux véhicules automobiles, $16^{\mathrm{e}}$ Congrès Français de Mécanique (CFM), Nice, France, 1 au 5 septembre 2003

[10] F. Chometon, P. Gillieron, Analysis of unsteady wakes by images processing in automotive aerodynamics, Congress FLUCOME, Tokyo, Japon, September 1997

Retrouvez nos articles sur le site : www.edpsciences.org/meca 